\title{
Projection effects in the spectra of early-type pulsating stars
}

\author{
P. Montañés Rodriguez and C. S. Jeffery
}

\author{
Armagh Observatory, College Hill, Armagh BT61 9DG, Northern Ireland, UK \\ e-mail: pmr@star.arm.ac.uk, csj@star.arm.ac.uk \\ Received 13 July 2000 / Accepted 7 June 2001
}

\begin{abstract}
The relation between the surface velocity of a pulsating star in the star's inertial frame and the apparent expansion velocity measured by a remote observer has been classically formulated in terms of a projection factor $p$. This factor depends on the relative limb darkening in continuum and spectral lines and may therefore be a function of composition. We have computed synthetic spectra to investigate the behaviour of $p$ and, indeed, of the apparent line profile for radially pulsating stars over a range of temperature and gravity with hydrogen-rich and hydrogen-poor atmospheres. We have subsequently derived values of $p$ suitable for the interpretation of velocity measurements of pulsating early type stars. Effective temperature has the most important influence on $p$ : a change of $10000 \mathrm{~K}$ in $T_{\text {eff }}$ introduces a change of 2-3 per cent with important consequences for measurements of stellar radii to an accuracy $<1 \%$.
\end{abstract}

Key words. stars: early-type - stars: pulsations - stars: atmospheres

\section{Introduction}

The expansion of a pulsating stellar atmosphere introduces a Doppler shift to the absorption spectrum. As a consequence of stellar surfaces being approximately spherical rather than coplanar with the sky, the apparent Doppler shift is a convolution of the projected motion $\dot{r} \mu$ with the specific intensity $I(\mu, \lambda)$ over the surface of the star $(\mu=\cos \theta)$. Formally, the total flux can be written,

$F(\lambda)=2 \int_{0}^{1} I\left(\mu,\left(1+\frac{\dot{r} \mu}{c}\right) \lambda\right) \mu \mathrm{d} \mu$.

This projection effect causes a given absorption line to be-

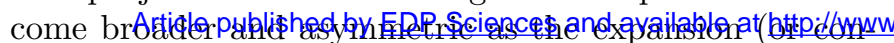
traction) velocity increases.

For an observer, the problem arises when an apparent expansion velocity, measured from the stellar absorption lines, needs to be converted into a expansion velocity in the star's inertial frame. This problem is most severe if the resolution of the observed spectrum, and its convolution with other line-broadening mechanisms (e.g. rotation, thermal, pressure, instrumental, acceleration) makes an inversion of Eq. (1) intractable. It has frequently been solved by recourse to a projection factor $p$ used to relate the apparent $v$ and true expansion velocity $\dot{r}$ of the stellar surface,

$v=-\dot{r} / p$.

Send offprint requests to: P. Montañés Rodriguez, e-mail: pmr@star.arm.ac.uk
Another problem arises with the spectroscopic measurement of stellar properties (e.g. effective temperature, surface gravity, chemical composition) where the line profile is of primary importance. Hence an ability to compute synthetic spectra which take Eq. (1) into account is important in any detailed analysis of radially pulsating stars.

\subsection{Previous work}

Early work was carried out by Eddington (1926) and Carroll (1928) for rotating and expanding atmospheres. After introducing an approximate expression for the limb darkening, both deduced a value $p=24 / 17$. Subsequently, Getting (1935) arrived at the same result by averaging line-of-sight velocity components over a limb-darkened aanda.org of htt
stellar disk.

This value was used for several decades in order to apply the Baade-Wesselink (1946) method to measure the radius of a pulsating star. In more modern times, Parsons (1972) adopted the more rigorous approach of computing the nett line profile from appropriately shifted profiles at different parts of the stellar disk. Previous calculations of this type, which had assumed Gaussian absorption profiles, had originally been made by Shapley \& Nicholson (1919). Parsons introduced the monochromatic emergent intensities $I(\mu, \lambda)$ computed from an appropriate model atmosphere in local thermodynamic equilibrium (LTE). The apparent velocity $v$ of the line was given by the effective line center measured at 0.6 of the central line depth, in order to simulate the operation of a Grant comparator. Parsons thus obtained a value $p=1.37-0.03 \gamma$ where

$\gamma=\dot{r} / w_{\frac{1}{2}}$ 
is the expansion velocity scaled by the half width (in velocity units) at half depth of the line (wavelength $\lambda$ )

$w_{\frac{1}{2}}=\frac{\Delta \lambda_{\frac{1}{2}} c}{\lambda}$.

Subsequently, Karp $(1973,1975)$ considered the effects of velocity gradients and weak and strong line profiles in moving atmospheres, while Hindsley \& Bell (1986) simulated the use of photoelectric-radial velocity spectrometers. They also reported a variation of $p$ due to the variation in limb-darkening in the absorption lines with stellar temperature. These values of $p$ vary between 1.31 $\left(T_{\text {eff }} \sim 4500 \mathrm{~K}\right)$ and $1.37\left(T_{\text {eff }} \sim 6500 \mathrm{~K}\right)$.

More detailed velocity structures are taken into account by Sasselov et al. (1989) who proved that an error of around 20-40 per cent in the radius given by the Baade-Wesselink method would arise when the asymmetry of the line is not considered. Sasselov \& Lester (1990) also showed that, for Cepheids, $p$ becomes larger in the infrared than in the visible by about 10 per cent, while Albrow \& Cottrell (1994) included a new treatment of macroturbulence and also reported $p \sim 10$ per cent larger than in previous studies.

\subsection{The helium star problem}

While most previous studies have been directed towards studies of Cepheids and similar stars, all have made the assumption that the stellar atmosphere has a normal hydrogen abundance. One question posed by Lynas-Gray et al. (1984) was whether Parsons' (1972) results for classical Cepheids could be applied directly to the pulsating helium star V652 Her. Since the opacities in the atmospheres of hydrogen-rich Cepheids and hydrogen-deficient pulsators are very different, as are their radii, the answer was not self-evident. Subsequent detailed studies of the projection effect in Cepheids and RR Lyraes (referred to already) shed no further light on this particular problem.

In this paper we report the development of techniques for computing synthetic spectra for radially expanding (or contracting) atmospheres. These have been applied, in particular, to models for early-type stars with extremely helium-rich surfaces for application to recent observations of pulsating helium stars V652 Her and BX Cir (Jeffery et al. 2001; Woolf \& Jeffery 2000). The behaviour of the absorption line profiles and the radial velocities measured therefrom have been examined in detail, and the results interpreted in terms of the projection factor $p$.

\subsection{Pulsation in other early-type stars}

The projection effect is closely related to that of limb darkening, which is essentially a function of the distribution of continuous opacity with optical depth in the stellar atmosphere. It is therefore expected to be a function of both wavelength and effective temperature, as well as chemical composition. Although the primary purpose of this investigation was to measure the projection effect for radially pulsating extreme helium stars, it was important to distinguish whether composition or effective temperature is the major factor. Thus, the case of radially-pulsating earlytype stars of normal composition has also been considered. Typical examples include the radial-mode $\beta$ Cepheids.

\section{Line profiles for expanding atmospheres}

\subsection{Model atmospheres and emergent intensities}

Line-blanketed plane-parallel models are calculated with the program STERNE, which assumes local thermodynamic, radiative and hydrostatic equilibrium. Full details have been given by Jeffery \& Heber (1992) and Jeffery et al. (2001). In order to investigate the effect of helium abundance in models of immediate interest, we used the models, with effective temperature $T_{\text {eff }}$, surface gravity $\log g$ and hydrogen abundance $n_{\mathrm{H}}$ (fractional by number) given by

a) $T_{\text {eff }} \overline{1} 5000 \mathrm{~K}, \log g=3.5, n_{\mathrm{H}}=0.90$,

b) $T_{\text {eff }} \overline{2} 5000 \mathrm{~K}, \log g=3.5, n_{\mathrm{H}}=0.01$,

c) $T_{\text {eff }} \overline{2} 5000 \mathrm{~K}, \log g=3.5, n_{\mathrm{H}}=0.90$, and

d) $T_{\text {eff }} \overline{2} 5000 \mathrm{~K}, \log g=5.5, n_{\mathrm{H}}=0.90$.

In all cases, the metal abundances $\left(n_{\mathrm{M}}\right)$ were solar and the helium abundances equal to $1-n_{\mathrm{H}}-n_{\mathrm{M}}$. In models b) and c) $T_{\text {eff }}$ and $\log g$ are close to those reported for pulsating helium stars V652 Her and BX Cir (Jeffery et al. 1999; Woolf \& Jeffery 2000). Models a) and d) were calculated to study the effect of an increase on $g$ and a decrease on $T_{\text {eff. }}$.

These models are used as input for the formal solution of the radiative transfer equations. This is carried out using the program SPECTRUM, our synthetic spectrum generator (Jeffery et al. 2001). One mode of operation of SPECTRUM provides the emergent intensities $I(\mu, \lambda)$ at specific cosine angles $\mu$, either for a given absorption line or for an entire region of spectrum including several hundred absorption lines.

The linelists for the $15000 \mathrm{~K}$ model are less complete than for the higher temperature models. This is not important for this paper because it is the average behaviour of a significant sample of absorption lines, rather than a detailed simulation of the spectrum, that is of interest.

We note that in the pulsating atmosphere of an earlytype or luminous star, the standard approximations (LTE, etc.) may break down, particularly in strong lines (nonLTE) or at minimum radius (shocks). In general, these lines are best avoided when making radial-velocity measurements by, for example, cross-correlation. In the case of V652 Her (Jeffery et al. 2001), a shock does occur at minimum radius, causing splitting in the strongest lines. However, for $90 \%$ of the pulsation cycle, the LTE planeparallel approximation has, so far, proved satisfactory.

\subsection{Integration of the emergent line profile}

This output is then used as input for the evaluation of Eq. (1) for a range of individual line profiles and synthetic 
spectra. Emergent intensities were calculated for 11 cosine angles $(\mu=1.0,0.9,0.8, \ldots, 0.1,0.01)$, and Eq. (1) was evaluated using Simpson integration. Individual lines included a range of weak and strong lines seen in the spectra of pulsating helium stars, including He I $4471 \AA$, He I $4438 \AA$, N II $4202 \AA$, N III $4510 \AA$, Si III $4553 \AA$, and Fe III $4396 \AA$.

After integration, the line profiles and synthetic spectra were normalized to the continuum. An illustration of the procedure, showing the normalized limb-darkened Doppler-shifted emergent intensity profiles at specific cosine limb-angles and the disk-integrated line profile is shown in Fig. 1.

\section{Analysis of results}

\subsection{Limb darkening}

It may be deduced from Eq. (1) that the main factor controlling the degree of asymmetry and hence the apparent line shift is effectively the ratio of limb darkening in the line to limb darkening in the continuum - in other words, how does the line strength change towards the stellar limb? We computed $I(\mu)$ in the continuum $I_{\mathrm{c}}$ and in the cores $I_{1}$ of a variety of absorption lines and we found that at $4471 \AA$ limb darkening is reduced by an increase in helium abundance, effective temperature or surface gravity (Fig. 2).

We also compared the limb darkening in the line $B_{1}(\mu)$ for both weak and strong absorption lines (Fig. 2, right panel). The limb-darkening has been normalized to $I_{\mathrm{c}}(\mu) / I_{\mathrm{c}}(1)$ to isolate the limb-darkening due to the lines, it can be defined as,

$B_{1}(\mu)=\frac{I_{1}(\mu) / I_{1}(1)}{I_{\mathrm{c}}(\mu) / I_{\mathrm{c}}(1)}$

If $B_{1}(\mu) \sim 1$ for all $\mu$, the line is undarkened. This is the case for line photons formed at small continuum optical depths, like the core of He I 4471 in the high-gravity model. The zero-velocity contribution to the profile integrated over the disk is thus significant, and dilutes the higher velocity contribution from points close to the stellar center. If repeated over the whole line profile, this would increase the line asymmetry and also the projection factor $p$.

If the line-to-continuous opacity ratio is reduced, line flux is formed at increasing depths. Then $B_{1}(\mu)$ can increase substantially towards small $\mu$ - the corollary is that for weak lines the relative absorption is reduced towards the limb, the high velocity contribution from $\mu \sim 1$ dominates, the line is more symmetric and $p$ should be smaller.

Figure 2 demonstrates that varying any atmospheric parameter has considerable influence on line darkening in the regions of strongest absorption, but that for moderately weak lines (e.g. Fe III), only the surface gravity has a significant effect.

\subsection{Projection factors: lines}

Similar pairs of a representative range of theoretical line profiles were computed for a range of hydrogen-rich models and for one hydrogen-poor model, and for a range of expansion velocities $\dot{r}$. The continuum was subtracted and the wavelengths were converted to a logarithmic scale. The cross-correlation function (ccf) of the projected line profile relative to the stationary line profile was calculated and transformed to velocity units. To find the position of the ccf maximum, we used a number of different methods including fitting Gaussians and parabolae to different fractions of the ccf maximum. It was apparent from these experiments that the method for measuring $v$ is crucial and that the determination of $p$ must be done in a manner consistent with the observational measurement of $v$.

Taking the position of the ccf maximum to represent $v$, values for the projection factor $p=-\dot{r} / v$ are shown in Table 1 and Fig. 3. These data were obtained from fitting a Gaussian to points within $30 \%$ of the ccf maximum.

The intrinsic profiles of different lines vary due to differences in the ion mass and the damping profiles. However these differences can be accounted for by considering the line half-width $\left(w_{\frac{1}{2}}\right)$. The apparent velocities for each line are shown in Fig. 3 in the form $p=-\dot{r} / v$ plotted against $\gamma=\dot{r} / w_{\frac{1}{2}}$. Line widths at half depth for the six studied lines in each model are shown in Table 2.

Most points, corresponding to different lines and different expansion velocities, fall approximately on the same curve. The major exception is the N III $4510 \AA$ line. Its position was found to be sensitive to the adopted abundance; it was possible to match the other line positions by increasing the nitrogen abundance. At the adopted abundance, this line is considerably weaker than all the others and, at large cosine limb-angles, the numerical quadrature of Eq. (1) became very inaccurate.

Other numerical errors in determining $p$ arise principally from sampling in the ccf. As the ccf becomes broader for larger $\dot{r}$ or for stronger lines, the number of points above some critical value in the ccf will increase. It is these points which are used to define the Gaussian which gives $v$. This ambiguity reflects the principal uncertainty in determining $p$, that is, the choice of method by which the radial velocity is measured. However, since most data fall on the same curve, we are satisfied that we understand the systematic effects prior to investigating the use of crosscorrelation techniques for large sections of spectrum.

\subsection{Projection factors: cross-correlation}

It is evident that the projection scale factor must be determined as far as possible by measuring the expansion velocity of the theoretical spectrum in exactly the same way as the observed spectrum is to be measured. Theoretical spectra were constructed by applying Eq. (1) to synthetic spectra computed in the interval $\lambda 4256-4980 \AA$, corresponding to new observations of V652 Her (Jeffrey et al. 2001). The theoretical intensity spectra were calculated 

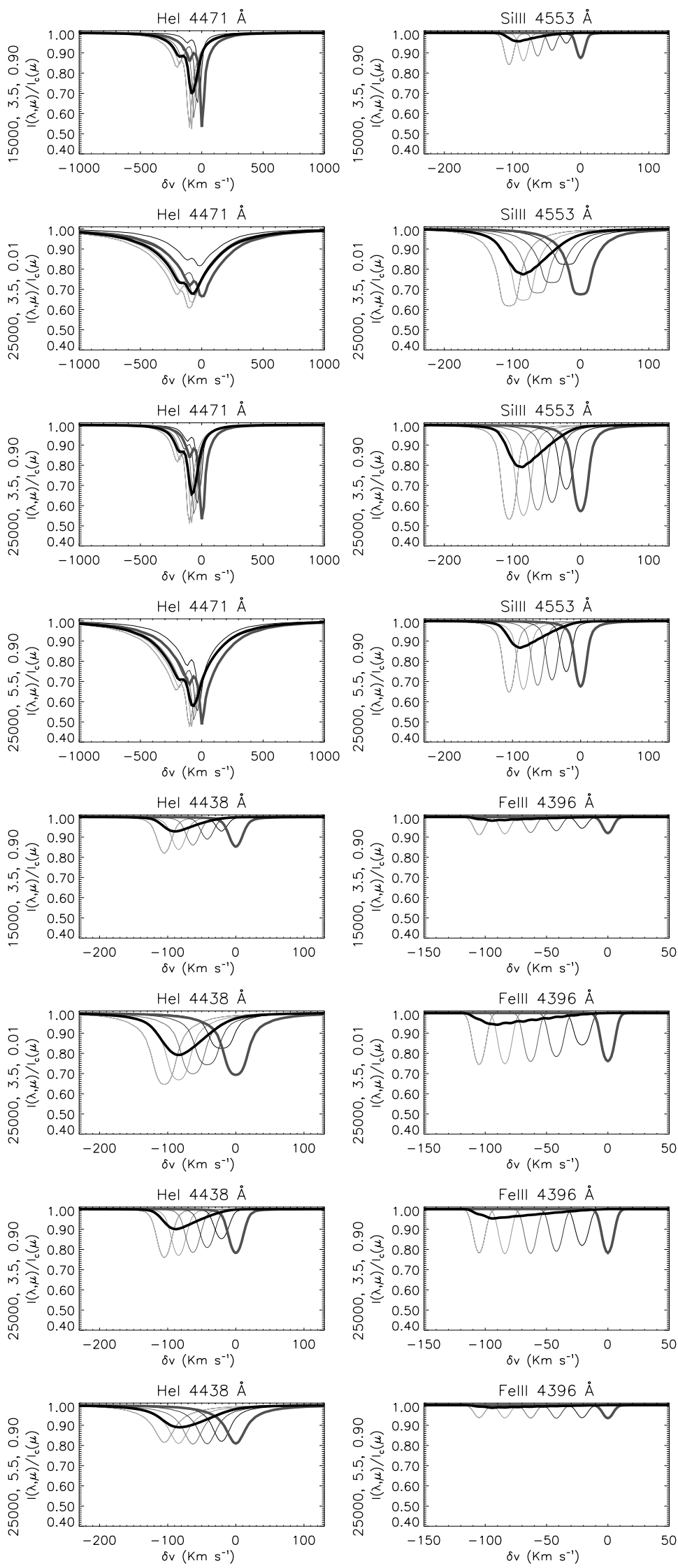

Fig. 1. Line profiles from four different expanding stellar atmospheres. Projected specific intensities at cosine limb-angles $\mu=1.0,0.8,0.6,0.4,0.2$ for He I $4471 \AA$, Si III $4553 \AA$, Fe III $4396 \AA$ and He I $4438 \AA$ for $\dot{r}=105 \mathrm{~km} \mathrm{~s}^{-1}$ for the models (left label) a), b), c) and d). In thicker line appear the intrinsic and apparent fluxes; note the asymmetry and blue shift of the apparent lines, both intrinsic and apparent lines have the same equivalent width. 

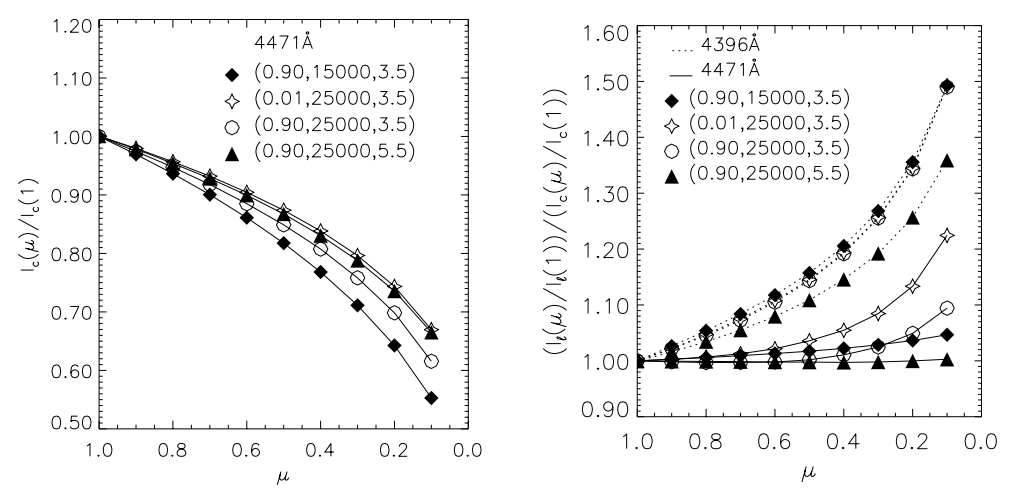

Fig. 2. The normalized emergent intensity $(I(\mu) / I(1))$ is shown (left) as a function of $\mu$ for the four different models at a wavelength of $4471 \AA$. In the right-hand panel, the limb-darkening in the line relative to the limb-darkening in the continuum is compared for both weak ( Fe III $4396 \AA$ ) and strong lines ( He I $4471 \AA$ ).
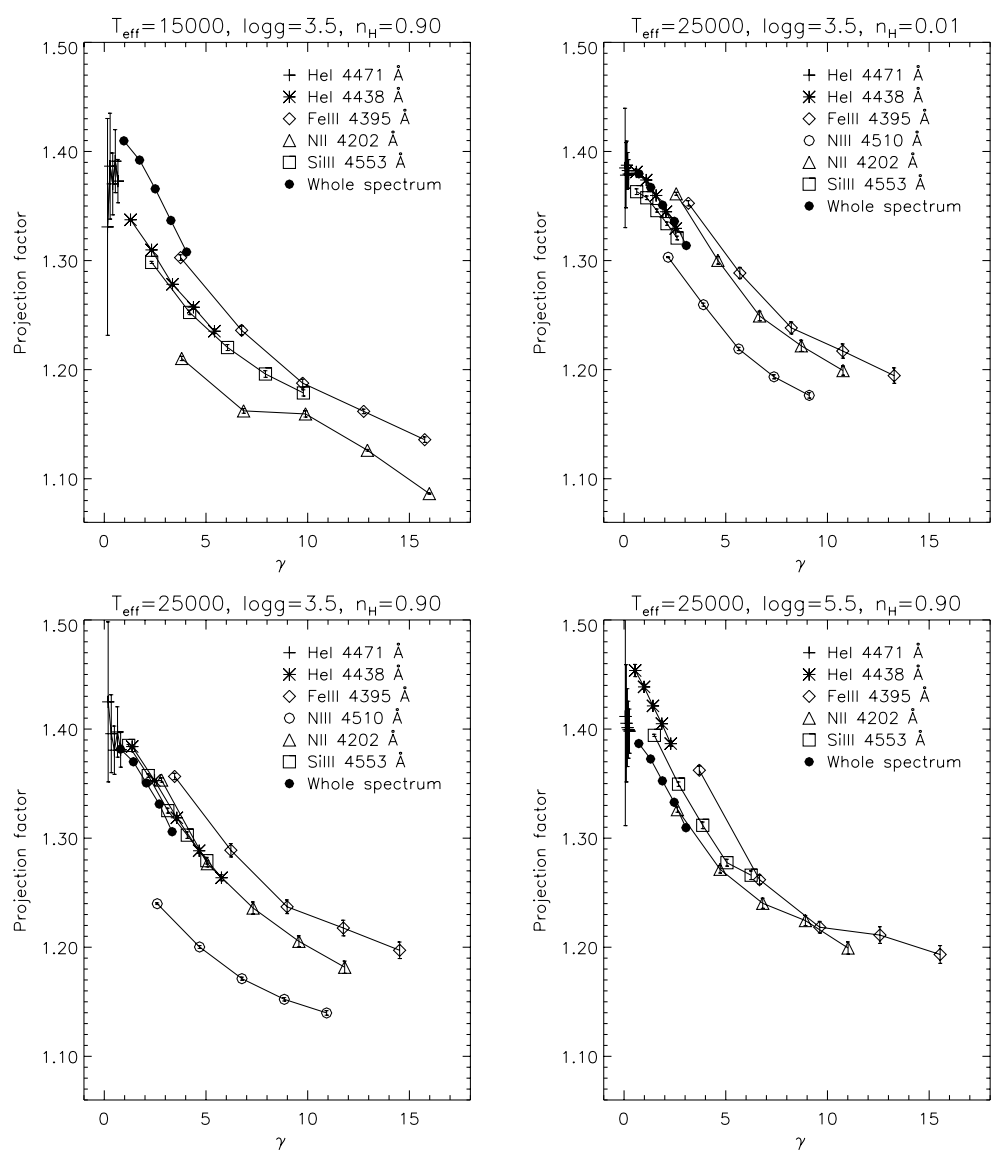

Fig. 3. Relationship between the projection factor $p$ and the scaled expansion velocity $\dot{r} / w_{\frac{1}{2}}$ for the cases of individual lines and the whole spectrum, for three solar composition models and a helium-rich model (top, right).

with microturbulent velocity $5 \mathrm{~km} \mathrm{~s}^{-1}$, and with a solar composition (H-rich model) and the composition derived for V652 Her (H-poor model, Jeffery et al. 1999). After evaluating Eq. (1) for expansion velocities $\dot{r}=$ $0,25,45,65,85$ and $105 \mathrm{~km} \mathrm{~s}^{-1}$, the resolution of the emergent spectra was degraded to $R=20000$, corresponding to the new observations.

Expansion velocities $v$ were measured from the entire spectrum by cross-correlation with the non-expanding models, after removing the broadest $\mathrm{H}$ and $\mathrm{He}$ I lines. The mean line-widths $2 \Delta \lambda_{\frac{1}{2}}$ of the spectra used in the calcula- tion of $\gamma$ were $0.7998 \AA, 1.0575 \AA, 0.9699 \AA, 1.0632 \AA$ for the models a), b), c) and d) respectively. The mean line widths obtained from the auto-correlation function represent an average over all lines selected and is only meaningful in terms of other quantities measured from the same region of the spectrum. It is dominated by the choice of lines included (e.g. blends, doublets, collisional broadening) and will generally be larger than for isolated weak lines. The mean line widths also reflect the fact that the spectra have been smoothed to simulate the instrumental broadening $(F W H M=0.4460 \AA)$. 
Table 1. Projection factors $p$ for individual absorption lines. Formal errors derived from the location of the ccf maximum are shown in parentheses (final digits). Expansion velocity $\dot{r}$ is expressed in $\mathrm{km} \mathrm{s}^{-1}$.

\begin{tabular}{|c|c|c|c|c|}
\hline Model & a) & b) & c) & d) \\
\hline$n_{\mathrm{H}}$ & 0.90 & 0.01 & 0.90 & 0.90 \\
\hline $\log g$ & 3.5 & 3.5 & 3.5 & 5.5 \\
\hline$T_{\text {eff }}$ & 15000 & 25000 & 25000 & 25000 \\
\hline$\dot{r}$ & \multicolumn{4}{|c|}{ He I $4471 \AA$} \\
\hline 25 & $1.331(99)$ & $1.385(55)$ & $1.425(73)$ & $1.412(100)$ \\
\hline 45 & $1.387(49)$ & $1.378(30)$ & $1.396(36)$ & $1.405(054)$ \\
\hline 65 & $1.370(28)$ & $1.387(22)$ & $1.381(22)$ & $1.401(036)$ \\
\hline 85 & $1.391(29)$ & $1.383(17)$ & $1.397(23)$ & $1.399(026)$ \\
\hline 105 & $1.373(20)$ & $1.379(13)$ & $1.381(16)$ & $1.398(020)$ \\
\hline$\dot{r}$ & \multicolumn{4}{|c|}{ He I $4438 \AA$} \\
\hline 25 & $1.337(1)$ & $1.381(3)$ & $1.384(0)$ & $1.454(6)$ \\
\hline 45 & $1.310(1)$ & $1.374(2)$ & $1.353(1)$ & $1.439(2)$ \\
\hline 65 & $1.278(1)$ & $1.360(1)$ & $1.319(2)$ & $1.421(2)$ \\
\hline 85 & $1.257(2)$ & $1.345(1)$ & $1.288(2)$ & $1.405(2)$ \\
\hline 105 & $1.235(2)$ & $1.329(2)$ & $1.264(3)$ & $1.387(2)$ \\
\hline$\dot{r}$ & \multicolumn{4}{|c|}{ Si III $4553 \AA$} \\
\hline 25 & $1.298(1)$ & $1.363(3)$ & $1.385(1)$ & $1.394(1)$ \\
\hline 45 & $1.252(2)$ & $1.358(2)$ & $1.357(2)$ & $1.349(2)$ \\
\hline 65 & $1.220(3)$ & $1.346(1)$ & $1.325(2)$ & $1.312(2)$ \\
\hline 85 & $1.196(3)$ & $1.334(2)$ & $1.303(3)$ & $1.277(3)$ \\
\hline 105 & $1.179(3)$ & $1.321(2)$ & $1.279(3)$ & $1.266(3)$ \\
\hline$\dot{r}$ & \multicolumn{4}{|c|}{ N III $4510 \AA$} \\
\hline 25 & - & $1.303(0)$ & $1.240(1)$ & - \\
\hline 45 & - & $1.260(1)$ & $1.200(1)$ & - \\
\hline 65 & - & $1.219(1)$ & $1.171(1)$ & - \\
\hline 85 & - & $1.193(2)$ & $1.152(2)$ & - \\
\hline 105 & - & $1.176(2)$ & $1.140(2)$ & - \\
\hline$\dot{r}$ & \multicolumn{4}{|c|}{ N II $4202 \AA$} \\
\hline 25 & $1.210(2)$ & $1.361(1)$ & $1.353(2)$ & $1.326(2)$ \\
\hline 45 & $1.162(2)$ & $1.300(3)$ & $1.277(3)$ & $1.271(3)$ \\
\hline 65 & $1.159(3)$ & $1.249(4)$ & $1.236(6)$ & $1.240(5)$ \\
\hline 85 & $1.126(1)$ & $1.222(5)$ & $1.205(5)$ & $1.224(5)$ \\
\hline 105 & $1.086(1)$ & $1.199(4)$ & $1.182(6)$ & $1.199(6)$ \\
\hline$\dot{r}$ & \multicolumn{4}{|c|}{ Fe III $4396 \AA$} \\
\hline 25 & $1.303(3)$ & $1.353(2)$ & $1.357(3)$ & $1.362(4)$ \\
\hline 45 & $1.236(4)$ & $1.289(5)$ & $1.289(6)$ & $1.262(4)$ \\
\hline 65 & $1.188(4)$ & $1.238(5)$ & $1.237(6)$ & $1.218(5)$ \\
\hline 85 & $1.162(2)$ & $1.217(7)$ & $1.218(7)$ & $1.211(8)$ \\
\hline 105 & $1.136(3)$ & $1.195(7)$ & $1.197(8)$ & $1.193(8)$ \\
\hline
\end{tabular}

The resulting projection factors are shown in Fig. 3 (filled circles) and Table 3.

Weak lines noted above will contribute in a minor way to the overall ccf and could possibly affect the measurement of $v$. However it appears from the co-incidence of $p$ measured from a large region of spectrum with $p$ measured from moderate to strong lines, that the nett contribution of weak lines is negligible.
Table 2. Line width at half depth $2 \Delta \lambda_{\frac{1}{2}}(\AA)$ calculated by measuring the width of the autocorrelation function of the line.

\begin{tabular}{ccccc}
\hline$n_{\mathrm{H}}$ & 0.90 & 0.01 & 0.90 & 0.90 \\
$\log g$ & 3.5 & 3.5 & 3.5 & 5.5 \\
$T_{\text {eff }}$ & 15000 & 25000 & 25000 & 25000 \\
\hline Line & \multicolumn{4}{c}{$2 \Delta \lambda_{\frac{1}{2}}$} \\
He I 4471 & 5.614 & 16.152 & 4.593 & 13.800 \\
He I 4438 $\AA$ & 0.626 & 1.456 & 0.561 & 1.641 \\
Fe III 4396 & 0.196 & 0.230 & 0.211 & 0.201 \\
N III 4510 & - & 0.345 & 0.288 & - \\
N II 4202 $\AA$ & 0.179 & 0.272 & 0.254 & 0.276 \\
Si III 4553 & 0.340 & 1.414 & 0.715 & 0.581 \\
\hline
\end{tabular}

Table 3. Projection factors for the entire spectrum (42564980 Å) (formal errors on last digit in parentheses).

\begin{tabular}{ccccc}
\hline Model & a) & b) & c) & d) \\
$n_{\mathrm{H}}$ & 0.90 & 0.01 & 0.90 & 0.90 \\
$\log g$ & 3.5 & 3.5 & 3.5 & 5.5 \\
$T_{\text {eff }}$ & 15000 & 25000 & 25000 & 25000 \\
\hline \multicolumn{5}{c}{$p$} \\
25 & $1.410(1)$ & $1.380(3)$ & $1.382(2)$ & $1.387(3)$ \\
45 & $1.392(1)$ & $1.367(2)$ & $1.370(2)$ & $1.373(2)$ \\
65 & $1.366(2)$ & $1.351(2)$ & $1.351(2)$ & $1.353(2)$ \\
85 & $1.337(2)$ & $1.336(3)$ & $1.331(2)$ & $1.333(3)$ \\
105 & $1.308(2)$ & $1.314(3)$ & $1.306(3)$ & $1.310(3)$ \\
\hline
\end{tabular}

Table 4. Fit coefficients for projection factors over the entire blue spectrum (4256-4980 ̊̊).

\begin{tabular}{ccccc}
\hline Model & a) & b) & c) & d) \\
\hline$n_{\mathrm{H}}$ & 0.90 & 0.01 & 0.90 & 0.90 \\
$\log g$ & 3.5 & 3.5 & 3.5 & 5.5 \\
$T_{\text {eff }}$ & 15000 & 25000 & 25000 & 25000 \\
$a_{0}$ & 1.447 & 1.402 & 1.410 & 1.414 \\
$a_{1}$ & -0.034 & -0.028 & -0.030 & -0.033 \\
\hline
\end{tabular}

In order to compare the results and to use them in correcting observed expansion velocities, we have constructed analytic fits. We find that for $1<\gamma \lesssim 4$, there is an approximately linear relation between $p$ and $\gamma$ of the form:

$p=a_{0}+a_{1} \gamma$.

\subsection{Projection factors: Temperature and gravity}

Table 4 also gives coefficients for Eq. (6) for model atmospheres with varying effective temperature and surface gravity.

It has been noted that $p$ should be greatest for the strongest lines; the largest values of $p$ are seen for He I lines in the high-gravity model (Fig. 3). Surface gravity 

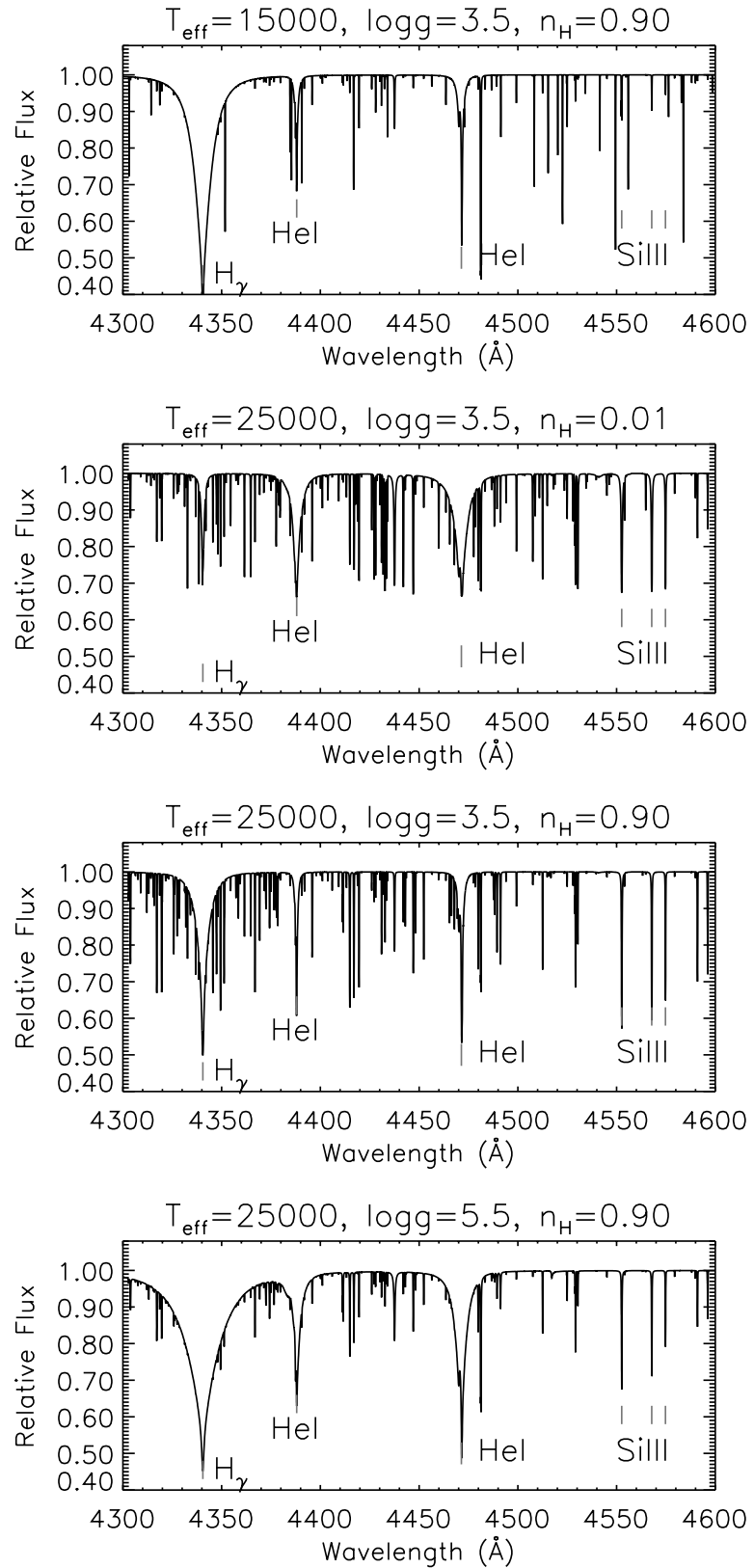

Fig. 4. Normalized synthetic spectra with no expansion used in the comparison of $p$ in the four model atmospheres considered. Note that all lines except $\mathrm{H} \gamma$, are stronger in the He-rich spectrum. The principal H, He I and Si III lines are marked.

has already been noted to reduce the line limb darkening $\left(B_{1}(\mu)\right)$ thus leading to the higher value of $a_{0}$ seen in Table 4.

Otherwise, values of $p$ obtained by cross-correlating the whole spectrum are reduced by an increase in helium abundance and effective temperature. The effect of composition may indeed be the smallest of all three as measured by the current models. The values of $p$ obtained from the whole spectrum measurements for $T_{\text {eff }} \overline{1} 5000 \mathrm{~K}$ are larger than those for all other absorption lines.

This may be because the lower $T_{\text {eff }}$ spectrum is dominated by weak lines of lower ionization potential ions. together with the smaller thermal broadening, the intrin-

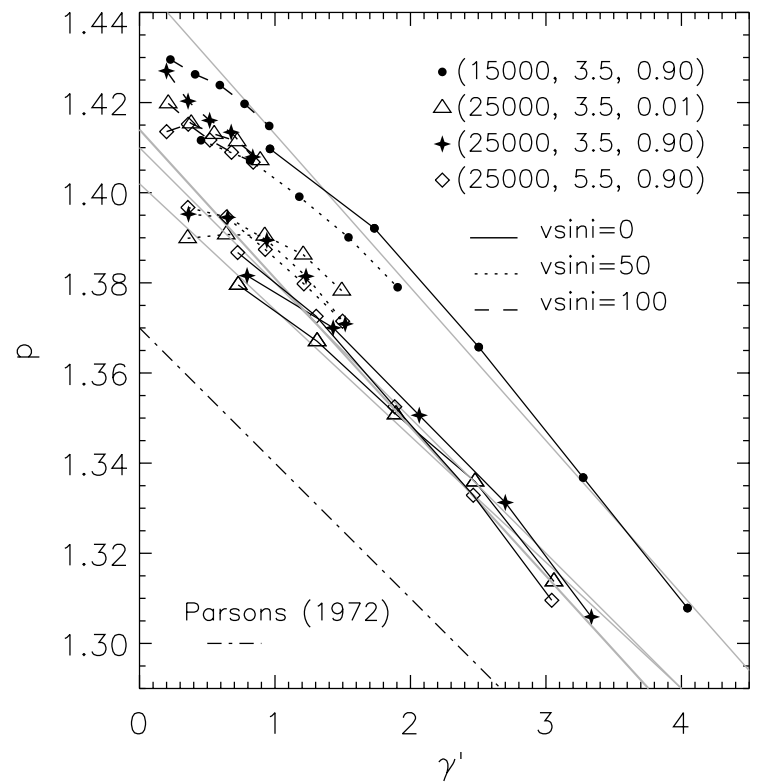

Fig. 5. Projection factors $p$ as a function of the modified $\gamma^{\prime}=\dot{r} / w_{\frac{1}{2}}^{\prime}$ for rotationally-broadened spectra with $v \sin i=$ $0,50,100 \mathrm{~km} \mathrm{~s}^{-1}$ and $\dot{r}=25,45,65,85,105 \mathrm{~km} \mathrm{~s}^{-1}$. The linear fits to $p(\gamma)$ from Eq. (6) and the result obtained by Parsons for cooler $(\sim 6000 \mathrm{~K})$ and lower gravity $(\log g \sim 1.5)$ model atmospheres is also shown.

sic line widths are smaller, on average, than the individual lines chosen for comparison. This should not have affected the overall result because of the use of $\gamma$. However, the change in balance of broad and narrow lines from, for example, light and heavy ions may have affected $p$ and $w_{\frac{1}{2}}$ in different ways. This appears to be borne out by the Fe III line which behaves similarly to the whole spectrum in Fig. 3 for the $15000 \mathrm{~K}$ model.

It is however clear that projection factors for all these models are higher than those normally used for the much cooler classical Cepheids (Parsons 1972).

\subsection{Rotation}

An investigation of the effect of rotation was carried out in which the integrated emergent spectra of the previous section were convolved with a rotation broadening function (Unsöld 1955) for projected rotation velocities $v \sin i=0,50,100 \mathrm{kms}^{-1}$. This has already been demonstrated by Duval \& Karp (1978) who showed how a small amount of rotation may make an asymmetric line profile even more asymmetric. The consequence is that the projection factor is increased with $v \sin i$.

For practical purposes it may be better to consider $\gamma^{\prime}=\dot{r} / w_{\frac{1}{2}}^{\prime}$ where $w_{\frac{1}{2}}^{\prime}$ is the mean line half-width of the rotationally-broadened spectrum with expansion velocity $\dot{r}=0$ (Fig. 5). Even in this framework, the effect of rotations is unclear, and some correction will be required in order to apply Eq. (6). 


\section{Conclusions}

The aim of this paper was to investigate the rôle of chemical composition on the projection factor relating the apparent and true radial velocities in radially pulsating stars, with particular reference to the case of B-type helium stars. We have calculated appropriate profiles for a range of spectral lines in both hydrogen-rich and helium-rich stellar atmospheres, and have simulated contemporary cross-correlation measurements using synthetic spectra. The well-known dependence of the projection factor on the width of the spectral line being used has been reproduced. The use of a large region of spectrum gives results consistent with individual line profiles when a mean line profile is used to normalize the expansion velocities. The effect of rotational broadening can also be taken into account if the line width can be measured at a phase when the star is not expanding or contracting.

Variations in $T_{\text {eff }}$ have the most significant influence on $p$, being $\sim 3 \%$ greater than Parson's values for a $25000 \mathrm{~K}$ star and $\sim 5 \%$ greater at $T_{\text {eff }}=15000 \mathrm{~K}$. After normalisation for the intrinsic line width, the influence of gravity, rotation and composition is an order of magnitude smaller than this. Although modest, the $T_{\text {eff }}$ effect is highly significant when direct measurements of stellar radii seek to achieve accuracies of $1 \%$ or better since, using Baade's method, $R \propto p$. Jeffery et al. (2001) achieved $\delta R / R=0.003$ in their study of $\mathrm{V} 652 \mathrm{Her}$. Even moderate resolution studies $(\delta R / R<0.10)$ may be compromised by a systematic error of this magnitude.

The factor $p$ to be adopted should always be determined from simulations that mimic the observational procedure as closely as possible. If, however, we had determined $p$ from simulations of the SiII line (Table 1) but had measured radial velocities by cross-correlating blue-optical spectra similar to those described in Sect. 3.3, we would have found $p$ and hence $R$ too small by $\sim 3 \%$. Choosing an arbitrary single line to determine $p$ could lead to errors in $R$ of up to $10 \%$ given the same ccf measurement of $v$. We have determined linear relations which should be appropriate for cross-correlation measurements of pulsating B stars observed in the blue-optical with a resolution $R \sim 20000$.

Acknowledgements. This research is supported by a grant to the Armagh Observatory from the Northern Ireland Department of Culture, Arts and Leisure. We are grateful to the referee whose valuable suggestions substantially improved this paper.

\section{References}

Albrow, M. D., \& Cottrell, P. L. 1994, MNRAS, 267, 548

Carroll, M. A. 1928, MNRAS, 88, 548

Duval, P., \& Karp, A. H. 1978, ApJ, 222, 220

Eddington, A. S. 1926, The Internal Constitution of the Stars (Cambridge University Press), 185

Getting, I. A. 1935, MNRAS, 95, 141

Hill, P. W., Kilkenny, D., Schönberner, D., \& Walker, H. J. 1981, MNRAS, 197, 81

Hindsley, R., \& Bell, R. A. 1986, ASP Conf. Ser., 98, 881

Jeffrey, C. S., \& Heber, U. 1992, A\&A, 206, 133

Jeffrey, C. S., Hill, P. W., \& Heber, U. 1999, A\&A, 346, 491

Jeffrey, C. S., Woolf, V. M., \& Pollacco, D. 2001, A\&A, submitted

Karp, A. H. 1973, ApJ, 180, 895

Karp, A. H. 1975, ApJ, 201, 641

Lynas-Gray, A. E., Schönberner, D., Hill, P. W., \& Heber, U. 1984, MNRAS, 209, 387

Parsons, S. B. 1972, ApJ, 174, 57

Sasselov, D. D., Lester, J. B. \& Fieldus, M. S. 1989, ApJ, 337, L29

Sasselov, D. D., \& Lester, J. B. 1990, ApJ, 362, 333

Shapley, H., \& Nicholson, S. B. 1919, Proc. Nature, Acad. Sci., 5,417

Unsöld, A. 1955, Physik der Sternatmosphären, 2nd ed. (Berlin Springer-Verlag)

Wesselink, A. J. 1946, Bull. astr. Inst. Netherlands, 10, 91

Woolf, V. W., \& Jeffrey, C. S. 2000, A\&A, 358, 1001 